\title{
LINEAR DISCRIMINANT FUNCTION ANALYSIS OF ACOUSTIC EMISSION SIGNALS FOR CUTTING TOOL MONITORING
}

\author{
Elijah KanNatey-Asibu and Erdal Emel \\ Department of Mechanical Engineering and Applied Mechanics, The University of Michigan, \\ Ann Arbor, MI 48109, U.S.A.
}

(Received February 1987, accepted June 1987)

\begin{abstract}
A principal setback to automation of the machining process is the inability to completely monitor the condition of the cutting tool in real time. Whereas several of the techniques developed to date are useful in specific applications, no universally applicable sensor is yet available.

Acoustic emission is one of the most promising techniques to be recently developed for on-line cutting tool monitoring. However, signal analysis is still an area that requires futher investigation to enhance the potential of acoustic emission. For this purpose, frequency-based pattern recognition concepts using linear discriminant functions have been used in analysing acoustic emission signals generated during machining to distinguish between different signal sources, specifically chip formation, tool fracture, and chip noise. Five features were used for classification in the frequency range of $100 \mathrm{kHz}$ to $1 \mathrm{MHz}$, with each feature consisting of a $20 \mathrm{kHz}$ bandwidth, and were selected using the class mean scatter criterion. The coefficients of the discriminant functions were obtained by training the system using signals generated by each of the sources of interest. An AISI 1018 steel was machined using a titanium carbide-coated cutting tool. Cutting speeds ranged from 200 to $800 \mathrm{ft} / \mathrm{min}(1$ to $4 \mathrm{~m} / \mathrm{sec}$ ) with feed rates of 0.0005 to $0.0075 \mathrm{in} / \mathrm{rev}$ $(0.0133 \mathrm{~mm} / \mathrm{rev}$ to $0.191 \mathrm{~mm} / \mathrm{rev})$ and depth of cut 0.17 in $(4.32 \mathrm{~mm})$. The results show a successful classification rate of $90 \%$ for tool breakage, while those for chip formation and chip noise were 97 and $86 \%$ respectively.
\end{abstract}

\section{INTRODUCTION}

The machining process constitutes a significant component of manufacturing operations (systems) and, quite often, leads to a bottleneck on the factory floor. The importance of the process stems from the fact that most primary processes are incapable of producing parts in final form and with the desired surface finish and texture. Whereas most machining processes can be implemented at relatively high production rates, especially with the continued development of machines and cutting tools capable of ultra high cutting speeds, such high production speeds often result in increased downtime due to frequent failure of the cutting tool either by wear or breakage. The need for continuous monitoring of the state of a cutting tool cannot therefore be over-emphasized, especially in an automated factory. However, the solution to the problem of real-time tool condition monitoring has been an elusive one in a universal sense, even though a variety of techniques have been developed that are useful in specific applications. Excellent reviews of these techniques have been made by Cook et al. [1], Micheletti et al. [2], Tlusty and Andrews [3] and Birla [4].

Acoustic emission (AE) is becoming increasingly important as a tool for monitoring several aspects of manufacturing processes. Basic research pertaining to the use of $\mathrm{AE}$ in investigating fundamental aspects of the metal cutting process have been highly 
successful with significant advances being made in the past few years [5-16]. Acoustic emission refers to the elastic stress waves generated as a result of the rapid release of strain energy within a material due to a rearrangement of its internal structure [8].

Advantages of $\mathrm{AE}$ as a tool wear sensing technique include

1. Its adaptability to computer control;

2. Its generation by processes in the cutting zone, including the chip-tool interface and the tool flank, which have a direct influence on tool wear;

3. The ability to detect signals from the cutting process away from the process zone, thus reducing the risk of instrumentation damage from the cutting environment;

4. The high frequency content of the emission signal, which is well beyond the frequency range of noise from machine tool dynamics and extraneous sources.

Recent efforts have resulted in significant advances in our understanding of the phenomenon of AE generation during metal cutting. However, substantial research is still necessary for a successful application in industry. One problem area is the need to distinguish between signals from different sources during cutting. Some of the significant signal sources that have been identified include

1. Those related to chip formation and tool wear (from the shear zone, tool-chip interface, and tool-work interface);

2. Those due to tool chipping and breakage;

3. Those resulting from the formed chip.

Careful monitoring of the signals from each of these sources provides very useful information about the cutting process and tool condition. The difficulty arises under normal cutting when all the sources can be active. It is then necessary to identify or distinguish between the various signals before further information can be obtained on the state of each source. For this, the use of a linear discriminant function based pattern recognition analysis was suggested by Kannatey-Asibu [10] and has also been implemented to detect chip breakage using event rate sensing by Dornfeld et al. $[12,15]$. This paper presents results obtained using frequency-based analysis of tool wear, breakage, and chip noise.

\section{BACKGROUND ANALYSIS}

Implementing a pattern recognition system requires three essential steps [17]

1. Sampling or scanning the input signal to produce the pattern space.

2. Extraction of the features, which often involves transformation of the signal from the pattern into the feature space, from which useful information can be obtained, and subsequent reduction of the data size.

3. Classification of the feature space to permit identification of the individual signal sources (classes).

The Bayesian decision rule forms the basis for the design of optimum classifiers. In the general case, it requires an estimate of the conditional density function of the data. However, for data with a Gaussian distribution and equal covariance matrices for the individual classes, the $a$ posteriori probability reduces to a linear discriminant function, simplifying the process of classification. Unfortunately, most real-life data are not normally distributed, and for these cases, sub-optimal linear classifiers can be designed using the least-mean-square error approach, the analytical basis for which is briefly presented in the following paragraphs as background information.

We consider sampled time series data (or pattern) represented as

$$
\mathbf{X}=\left[x_{1}, x_{2}, \ldots x_{N}\right]
$$


which is transformed into a feature space, giving

$$
\mathbf{Y}=\left[y_{1}, y_{2}, \ldots y_{N}\right] \text {. }
$$

The purpose of the transformation is to restructure the original data into a form that will locate patterns belonging to specific classes into distinct groupings that are easy to distinguish from other classes. It can be shown that the optimum transform that produces uncorrelated features, with respect to the mean-square error criterion, is the KarhunenLoeve transform. However, we base our analysis on the Fourier transform even though it may not yield the best features for classification (since it results in non-zero off-diagonal elements of the transform domain covariance matrix), and thus use the spectral components of the signal as features for two reasons

1. To permit a more useful physical interpretation of the results.

2. The Karhunen-Loeve transform is based on the covariance matrix of the data and, thus, cannot be implemented using a fast algorithm. This is an important consideration since the response speed of the sensor is a principal consideration in a machine tool monitoring system.

The Fourier transform for an analog signal is defined for an infinite time period. The use of discrete data of finite size, $N$, for transformation involves, in effect, the multiplication of an infinite data series by a rectangular window. This is equivalent to a convolution of the Fourier transforms of the infinite data and the window.

For a rectangular window defined as

$$
h(t)= \begin{cases}1 & \text { for }|t|<\tau / 2 \\ 0 & \text { elsewhere }\end{cases}
$$

the corresponding transform is the sinc function

$$
H(f)=\frac{\tau \sin (\pi f \tau)}{\pi f \tau}
$$

which has spurious peaks or sidelobes that decay slowly, as shown in Fig. 1. The end result is a distortion of the transform of the original signal, and to minimise its effect, we use a large data set. Increasing the data size reduces the width of the main lobe of the rectangular transform and, consequently, the extent of distortion. However, a larger data size or dimensionality also increases the resolution of the spectrum, making subsequent computations more involved. A prime consideration in the development of any

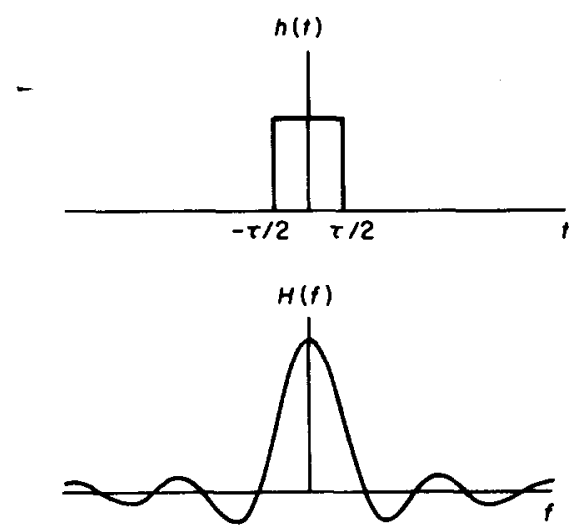

Figure 1. Fourier transform of a rectangular pulse. 
tool condition sensor is the speed of response, since it determines how soon the cutting tool can be retracted before any damage is done and is especially critical in ultra high speed machining. To improve the computational efficiency, we reduce the data size or dimensionality by eliminating the spectral components that convey the least information about the system. This stage of analysis is referred to as data reduction, and we use the class mean scatter criterion for feature selection. First, we determine the feature mean for each class, $\overline{\mathbf{Y}}_{\mathbf{i}}$

$$
\overline{\mathbf{Y}}_{\mathbf{i}}=\frac{1}{\boldsymbol{M}_{i}} \sum_{k=1}^{M_{i}} \mathbf{Y}_{\mathbf{i k}}
$$

where $M_{i}=$ the number of patterns in class $C_{i}$.

The overall system mean, $\overline{\mathbf{Y}}$, is then determined as

$$
\overline{\mathbf{Y}}=\sum_{i=1}^{c} p_{i} \overline{\mathbf{Y}}_{\mathbf{i}}
$$

where $p_{i}=$ the $a$ priori probability of class $C_{i}$ and $C=$ the number of classes.

We then obtain the scatter within each class by calculating the frequency domain covariance matrix as

$$
\mathbf{R}_{\mathbf{i}}=\frac{1}{M_{i}} \sum_{k=1}^{M_{i}}\left(\mathbf{Y}_{\mathbf{i k}}-\overline{\mathbf{Y}}_{\mathbf{i}}\right)\left(\mathbf{Y}_{\mathbf{i k}}-\overline{\mathbf{Y}}_{\mathbf{i}}\right)^{T}
$$

giving an overall system covariance matrix of

$$
\mathbf{R}=\sum_{i=1}^{c} p_{i} \mathbf{R}_{\mathbf{i}}
$$

The scatter between the individual classes is defined as

$$
\mathbf{R}_{\mathbf{c}}=\sum_{i=1}^{C} p_{i}\left(\overline{\mathbf{Y}}_{\mathbf{i}}-\overline{\mathbf{Y}}\right)\left(\overline{\mathbf{Y}}_{\mathbf{i}}-\overline{\mathbf{Y}}\right)^{T}
$$

From which the feature selection criterion is defined as

$$
\mathbf{Q}=\frac{\mathbf{R}_{\mathbf{c}}(j, j)}{\mathbf{R}(j, j)}
$$

where $\mathbf{R}_{\mathbf{c}}(j, j)$ and $\mathbf{R}(j, j)$ are the $j$-th diagonal elements of the covariance matrices $\mathbf{R}_{\mathbf{c}}$ and $\mathbf{R}$ respectively.

Since the objective is to minimise the scatter within the individual classes, while maximising the scatter between the classes, the desired number of features with maximum $Q$ values are selected as features. The number of features used is very important, since in addition to reducing computation efficiency, a high dimensionality requires a large number of experimental data sets for training the system and developing the classifier. This is because the adequate number of training data sets has to be about four or more times the number of features [18]. However, an excessive number of sets adds to the cost without necessarily improving the system performance. On the other hand, a small number of features provides an insufficient description of the system, since too small a training set will result in inadequate evaluation of the classifier weighting functions. For this work, it was found that five features provided the best performance.

With the desired number of features known the data sets could be represented in a five-dimensional feature space to show their relationship to each other. For ease of graphical representation, however, two of these features obtained from the data, specifically the 110 and $990 \mathrm{kHz}$ band frequencies, were selected and are plotted in a 
two-dimensional feature space in Fig. 2; these features will be discussed in a subsequent section. Even though the plot in Fig. 2 is limited to two features, it is obvious that there is an overlap between the various classes. Thus with a basic linear decision-making process, there can be a substantial amount of misclassification, where signals belonging to one class are erroneously assigned to another. This misclassification can be minimised by further transformation of the patterns from the feature space to a decision space using a transform that relocates the patterns such that all patterns belonging to a specific class are positioned in a region distinct from other classes, resulting in a decision space that facilitates classification.

For each class, $C_{i}$, we define a point, $V_{i}$, in the decision space around which we cluster all patterns belonging to $C_{i}$. Since there is still the likelihood of some misclassification even after this step, the objective of the transformation will be to minimise the decisionmaking errors that will subsequently result. Let us denote the transformation matrix for $C_{i}$ by $\mathbf{T}_{\mathbf{i}}$. Then a pattern $\mathbf{Y}_{\mathbf{i j}}$ in the feature space becomes

$$
S_{i j}=T_{i} Y_{i j}
$$

in the decision space.

Since $S_{i j}$ will not necessarily be located exactly at $V_{i}$, the error vector associated with that pattern after transformation is

$$
\varepsilon_{i j}=S_{i j}-V_{i}=T_{i} Y_{i j}-V_{i} .
$$

For class $C_{i}$, the total mean square error vector is

$$
\varepsilon_{\mathrm{i}}=\frac{1}{M_{i}} \sum_{j=1}^{M_{i}}\left|\varepsilon_{i j}\right|^{2}
$$

By substituting equation (7a) into ( $7 b$ ) and differentiating with respect to $\mathbf{T}_{\mathbf{i}}$ to minimise the error, and summing over all three classes for this case, we get an overall transformation matrix:

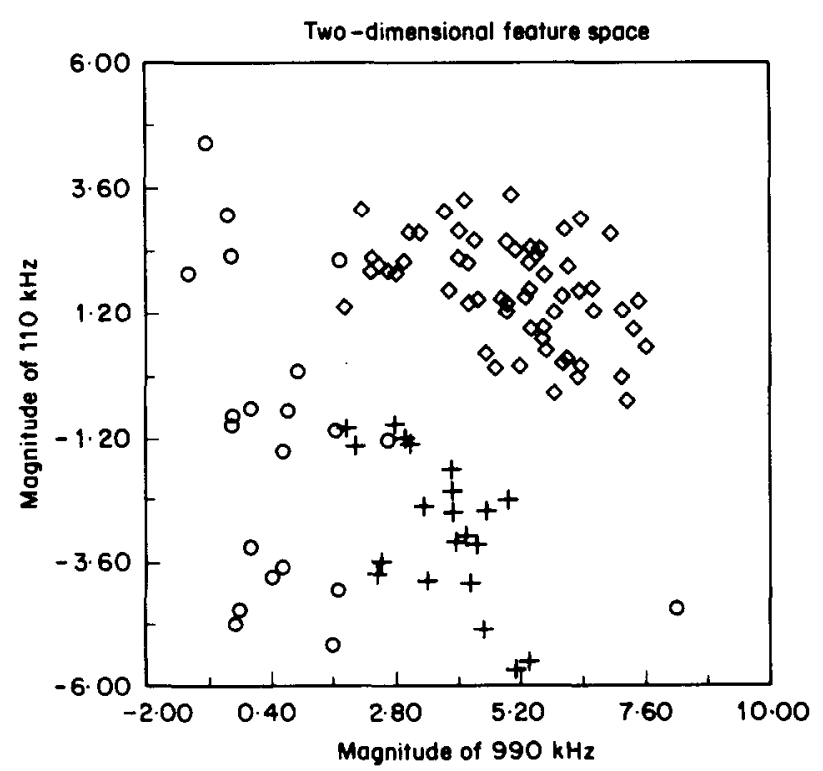

Figure 2. Feature space of acoustic emission signals from machining. $\diamond$, Chip formation; 0 , chip noise; + , tool breakage. 


$$
\mathbf{T}=\left[\sum_{i=1}^{3} \sum_{j=1}^{M_{i}} \frac{p_{i}}{M_{i}}\left(\mathbf{V}_{\mathbf{i}} \mathbf{Y}_{\mathbf{i j}}^{\mathrm{T}}\right)\right]\left[\sum_{i=1}^{3} \sum_{i=1}^{M} \frac{p_{i}}{M_{i}}\left(\mathbf{Y}_{\mathrm{ij}} \mathbf{Y}_{\mathrm{ij}}^{\mathbf{T}}\right)\right]^{\prime} .
$$

To classify an incoming signal, $\mathbf{S}_{\mathrm{ij}}$, we determine its distance from each of the predefined points, $V_{i}$, and assign it to the class to which it is closest. The distance, $d_{i}$, is given by

$$
d_{i}^{2}=\left\|\mathbf{S}_{\mathbf{i j}}-\mathbf{V}_{\mathbf{i}}\right\|^{2}, \quad i=1,2,3 .
$$

By expanding the right-hand side of equation (9) we can show that the minimum distance vector, $\mathbf{d}$, is obtained when the function

$$
\mathbf{g}=\mathbf{V T Y}
$$

is maximum. If we define the pre-selected points by unit vectors that result in a unit matrix for $\mathbf{V}$, then we have

$$
\mathbf{g}=\mathbf{T Y}
$$

with each row being the linear discriminant function, $g_{i}$, for a specific class, $C_{i}$, viz.

$$
g_{i}(\mathbf{Y})=w_{i 1} y_{1}+w_{i 2} y_{2}+\cdots+w_{i d} y_{d}-\Theta \quad i=1,2,3
$$

where $w_{i k}=$ weighting coefficient of the $k$-th feature for class $C_{i}$. For each sampled signal, the classifier will calculate the value of each discriminant function and assign the signal to the class with the maximum value.

The determination of elements of the transformation matrix $\mathbf{T}$ or the weighting coefficients of the discriminant functions requires training of the system. For that, we generate data under carefully controlled conditions that produce signals belonging to a particular class without any interference from signals of other classes.

\section{EXPERIMENTAL}

Data for training and testing the classifier was generated using a 30 h.p. CNC lathe machine. The workpiece was a cold-drawn AISI 1018 seamless steel tube, and the cutting tool a Valenite grade VN-8 titanium carbide coated insert, type TNMA-432. Cutting was done in the semi-orthogonal mode, with cutting speeds ranging from 200 to $800 \mathrm{ft} / \mathrm{min}$ ( 1 to $4 \mathrm{~m} / \mathrm{sec}$ ), feed rates of 0.0005 to $0.0075 \mathrm{in} / \mathrm{rev}(0.0133 \mathrm{~mm} / \mathrm{rev}$ to $0.191 \mathrm{~mm} / \mathrm{rev}$ ) and depth of cut of 0.17 in $(4.32 \mathrm{~mm})$. The specific conditions used are listed in Table 1 .

The acoustic emission transducer, a PAC WD-277, was mounted on the end of the cutting tool as shown in Fig. 3 and connected to a low-noise preamplifier with $40 \mathrm{~dB}$ gain. The preamplifier was positioned close to the transducer to minimise the influence of extraneous noise signals, since the original AE signals as they come out of the transducer are normally of rather low amplitude, on the order of 1 to $50 \mathrm{mV}$ and therefore highly susceptible to interference from extraneous signals. From the preamplifier, the signal goes to the signal processing unit, which has an adjustable gain amplifier that is set to a gain appropriate with the process under investigation. Since the present objective is the development of the signal processing technique, analysis of the signals is currently done off-line, first by recording the output of the signal processing unit on a $2 \mathrm{MHz}$ bandwidth AE recorder and subsequently playing back for analysis. On playback, the signal was passed through a signal gating unit where a $16.7 \mathrm{msec}$ length of the signal was clipped for sampling, with the recorder put in a pause mode at a desired location of the tape. To avoid saturation, the signal was then passed through an attenuator to reduce the amplitude 
TABLE 1

Experimental conditions

\begin{tabular}{ccc}
\hline $\begin{array}{c}\text { Cutting speed } \\
\mathrm{ft} / \mathrm{min}(\mathrm{m} / \mathrm{sec})\end{array}$ & $\begin{array}{c}\text { Feed rate } \\
\text { in } / \mathrm{rev}(\mathrm{mm} / \mathrm{rev})\end{array}$ & $\begin{array}{c}\text { Depth of cut } \\
\text { in }(\mathrm{mm})\end{array}$ \\
\hline & 0.0005 & \\
200 & $(0.0133)$ & \\
$(1.0)$ & 0.0075 & \\
& $(0.1905)$ & \\
& 0.0005 & 0.170 \\
400 & $(0.0133)$ & $(4.320)$ \\
$(2.0)$ & 0.0075 & \\
& $(0.1905)$ & \\
800 & 0.0005 & \\
$(4.0)$ & $(0.0133)$ & \\
\hline
\end{tabular}
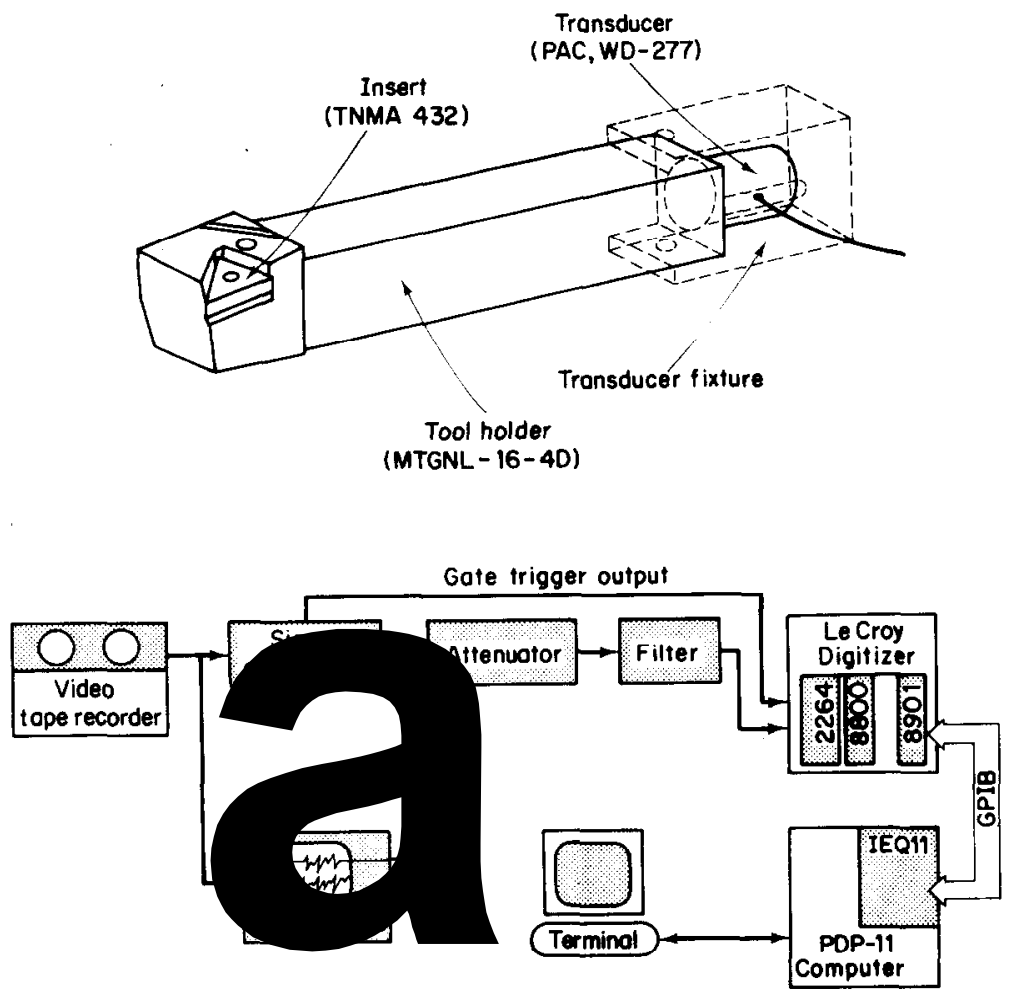

Figure 3. (a) Acoustic emission monitoring system-transducer mount. (b) Acoustic emission monitoring system-data acquisition set-up.

to a level compatible with the 8-bit high-speed waveform digitizer, which has a sampling rate capability of up to $4 \mathrm{MHz}$. The digitizer is controlled through a GPIB bus, and signal acquisition and storage is initiated when a post trigger signal is received by the digitizer from the computer. In using the GPIB bus, interface boards were required in both the computer and the digitizer unit along with a special purpose communications code.

Before sampling, the signal was first band-pass filtered with a $100 \mathrm{kHz}$ lower cutoff to eliminate low frequency noise signals and a $1 \mathrm{MHz}$ upper cutoff frequency to avoid 
aliasing at a sampling rate of $2 \mathrm{MHz}$. Due to computational limitations, only $4 \mathrm{~K}$ sampled data points were used for analysis, covering a time span of $2 \times 10^{-3} \mathrm{sec}$, with a resolution of $500 \mathrm{~Hz}$ in the spectral domain.

The regular machining signal was obtained from a portion of the signal that was essentially continuous and was observed to have no intereference from chips, while chip breakage samples were obtained from the burst signals that had periodicity indicative of chip breakage. Tool breakage signals were obtained from AE generated during fracture of pre-notched tools.

The original signal generated by the source undergoes some changes as it propagates through the structure and during transduction by the various instrumentation. Thus the
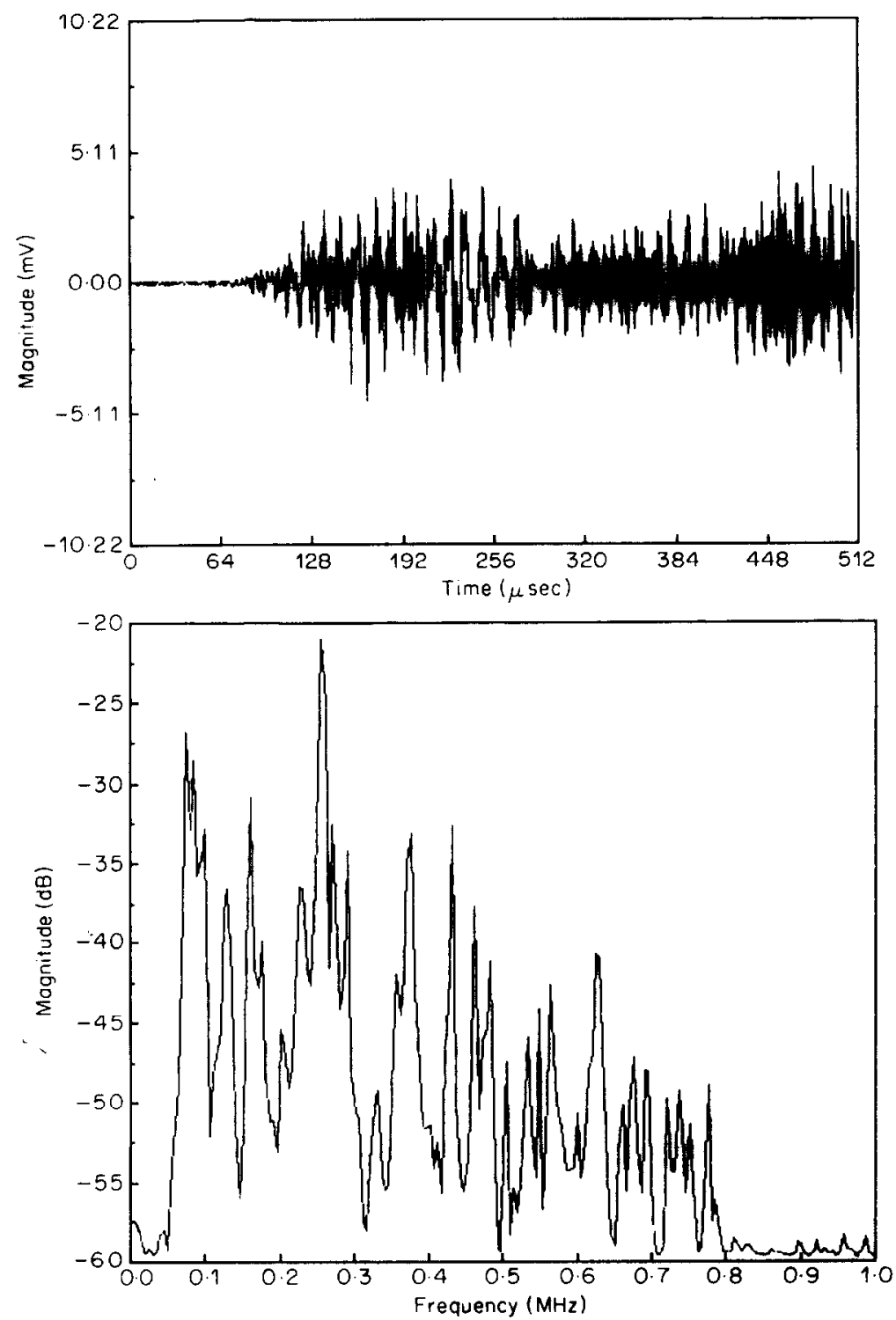

Figure 4. (a) Time domain signal of pencil lead breakage for calibration. (b) Frequency response of calibration signal. 
characteristics of the detected signal differs from that originally generated from the source. However, the original signal form can be obtained by deconvolution if the transfer function of the propagating medium and instrumentation is known, and this is obtained by calibrating the set-up. In this work, the propagating medium consists essentially of the cutting tool and holder. Calibration involved breaking a pencil lead of diameter $0.3 \mathrm{~mm}$ and length $3 \mathrm{~mm}$ at the tip of the cutting tool, with the equipment set up for experimental work. The spectrum of the recorded calibration signal was then deconvolved with the spectrum of the signal obtained during cutting to obtain the original signal characteristics. The time domain of the lead breakage and its corresponding spectrum are shown in Fig. 4.

\section{RESULTS AND DISCUSSION}

The analysis uses the spectral components of the acoustic emission signal as features for classification. The features were selected using the class mean scatter criterion discussed earlier, but the number used for the design was obtained by comparing the performance based on 3,5,10, and 20 features. All twenty features are shown ranked in Table 2, along with their computed values for the selection criterion. The results of classification for each set of features, shown in Table 3, indicate that five features produce the best performance. This is not necessarily a general rule, but results in this case, from the limited amount of training data available, which makes the larger feature sizes inappropriate for the classifier design. The specific features used for the classifier design, Table 2, are the $990,1000,970,110$, and $350 \mathrm{kHz}$ spectral components, arranged in descending order of importance. The poorer performance of the three-feature set compared to the five-feature set is due to the lack of adequate information in the three features to describe the system.

Before further discussing the results based on the analysis, we consider the visual differences between the signals from the various classes of interest. Figures 5 and 6 show

TABLE 2

Selected features

\begin{tabular}{crc}
\hline Rank & Feature & Q \\
\hline 1 & $990 \mathrm{kHz}$ & 1.416 \\
2 & $1000 \mathrm{kHz}$ & 1.075 \\
3 & $970 \mathrm{kHz}$ & 1.009 \\
4 & $110 \mathrm{kHz}$ & 0.992 \\
5 & $350 \mathrm{kHz}$ & 0.935 \\
6 & $950 \mathrm{kHz}$ & 0.833 \\
7 & $930 \mathrm{kHz}$ & 0.778 \\
8 & $330 \mathrm{kHz}$ & 0.761 \\
9 & $910 \mathrm{kHz}$ & 0.747 \\
10 & $890 \mathrm{kHz}$ & 0.728 \\
11 & $870 \mathrm{kHz}$ & 0.724 \\
12 & $430 \mathrm{kHz}$ & 0.648 \\
13 & $850 \mathrm{kHz}$ & 0.626 \\
14 & $530 \mathrm{kHz}$ & 0.560 \\
15 & $410 \mathrm{kHz}$ & 0.536 \\
16 & $710 \mathrm{kHz}$ & 0.532 \\
17 & $450 \mathrm{kHz}$ & 0.522 \\
18 & $550 \mathrm{kHz}$ & 0.511 \\
19 & $670 \mathrm{kHz}$ & 0.468 \\
20 & $690 \mathrm{kHz}$ & 0.400 \\
\hline
\end{tabular}


TABLE 3

Classification results for various features

\begin{tabular}{ccccc}
\hline $\begin{array}{c}\text { Feature set } \\
\text { dimension }\end{array}$ & Testing method & $\begin{array}{c}\text { Classifier performance (\%) } \\
\text { Chip } \\
\text { formation }\end{array}$ & $\begin{array}{c}\text { Chip } \\
\text { noise }\end{array}$ & $\begin{array}{c}\text { Tool } \\
\text { breakage }\end{array}$ \\
\hline \multirow{2}{*}{3} & Resubstitution & 83 & 95 & 38 \\
& Leave one out & 82 & 95 & 24 \\
5 & Resubstitution & 98 & 95 & 90 \\
& Leave one out & 97 & 86 & 90 \\
10 & Resubstitution & 98 & 90 & 90 \\
& Leave one out & 98 & 86 & 81 \\
20 & Resubstitution & 97 & 95 & 90 \\
& Leave one out & 95 & 71 & 76 \\
\hline
\end{tabular}

both the time and spectral domain plots of $\mathrm{AE}$ signals from normal machining and tool fracture, respectively. We only show two types for simplicity. The machining signal was obtained from a portion of a continuous signal that was observed to have no interference from chips and it is apparent that the regular machining signals are continuous, with any individual events being so close together as to overlap and thus be indistinguishable. On the other hand, the tool fracture signals consist of distinct burst signals, indicative of specific events.

It can also be observed from the diagrams that the spectrum for the chip formation signal shows intense activity in the lower frequency ranges of about 100 to $700 \mathrm{kHz}$. On the other hand, that for tool breakage is spread over a wide frequency range, with significant signal magnitudes up to $1 \mathrm{MHz}$. This difference in behaviour can be explained by the fundamental mechanisms that form the basis for each process. The chip formation process results essentially from a shearing action associated with dislocation motion, from which the rate at which energy is released (i.e. the risetime of a waveform generated by dislocation motion) is slow, resulting in characteristic frequencies concentrated in the lower frequency range. On the other hand, the sudden activity associated with brittle fracture produces energy that is more representative of an impulse, with a resulting spectrum over a wide frequency range. These basic differences in characteristics indicate that with the appropriate analysis, it is possible to identify the various forms of signal sources.

A more convenient perception of the differences between the classes can be obtained from the data in the feature space. As noted earlier, since there are five features, graphical representation of the entire feature space will not be feasible. Thus, we select two of the best features, viz., the 110 and $990 \mathrm{kHz}$ spectral components for plotting a two-dimensional feature space, Fig. 2. Even though this is not representative of the overall feature space, it illustrates the distinct differences and clustering of signals belonging to individual classes. It is also apparent from the figure that whereas signals generated by tool breakage and tool wear (or chip formation) form closely knit clusters, chip noise tends to be more scattered. Thus tool breakage can be identified from other machining signals much more easily, and with greater reliability than signals generated by the chip, which are more likely to induce misclassification. The greater variance in the chip signals is due to the varied nature in which chips can generate AE signals. Since deformation in the shear zone results in work-hardening of the chip, its breakage is, in effect, similar to brittle fracture, and the extent of similarity depends on the initial hardness of the workpiece, as well as the deformation. Also, the unbroken chip tends to rub against either the 

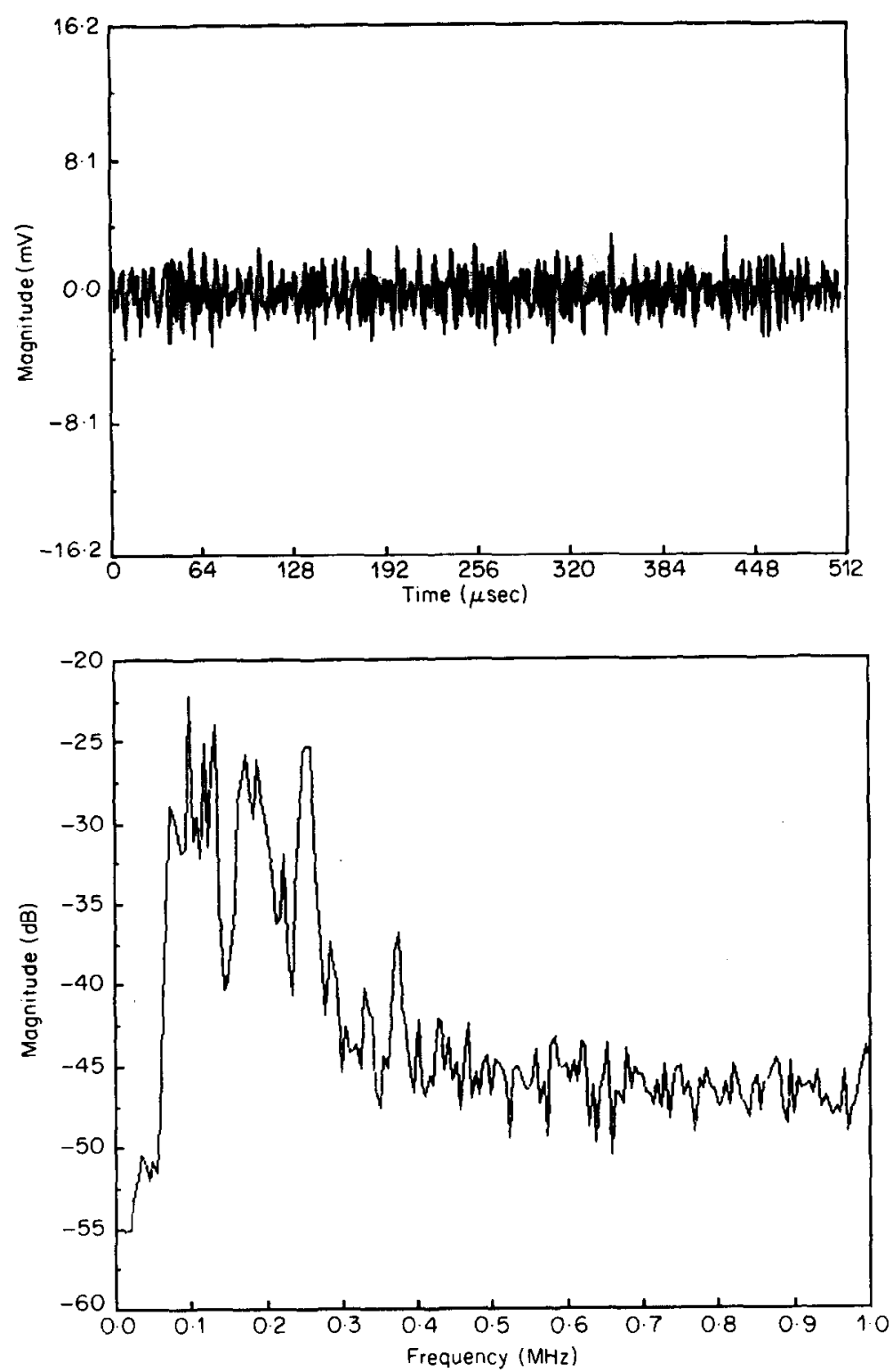

Figure 5. (a) Acoustic emission signal from chip formation. (b) Spectrum of chip formation signal.

workpiece or cutting tool, and furthermore, even an efficiently broken tool might rub against the workpiece before falling off. The result of the latter effects is the generation of signals with a lower frequency content which, when combined with the breakage effect, produces signals that are widely scattered in the feature space.

The process of classification is better visualised by representing the patterns in the decision space. The axes of the decision space constitute the individual classes. In this work, we consider the three signal sources, viz., chip formation, tool breakage, and ship noise, as the classes of interest. Even though for the three-class system, a three-dimensional decision space would be the most appropriate representation, again difficulties associated with visualisation make the two-dimensional plot preferable. Figure 7 shows the three combinations based on the three classes of interest. The distinct separation between the 

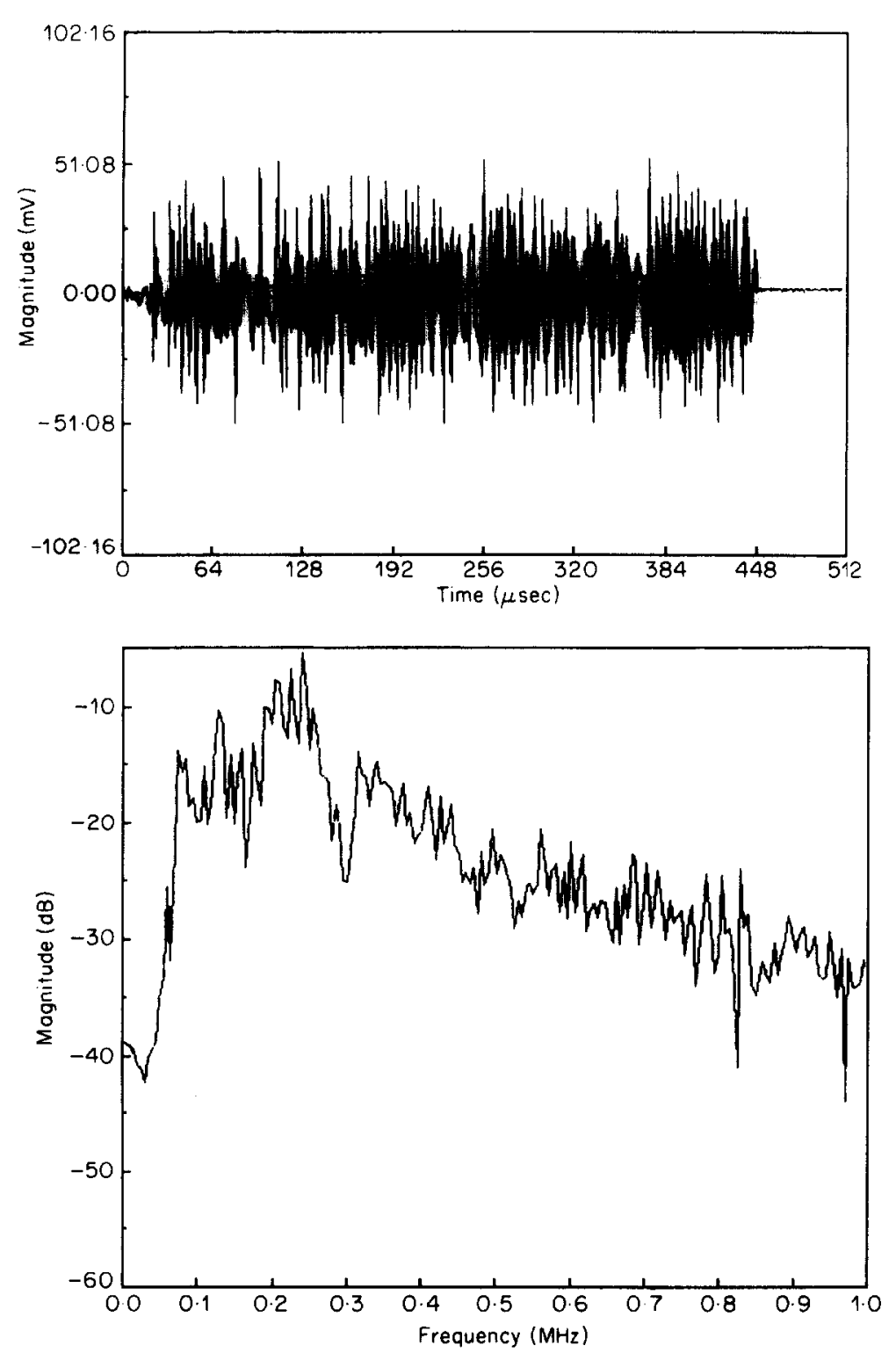

Figure 6. (a) Acoustic emission signal from tool breakage. (b) Spectrum of tool breakage signal.

various classes is more evident in these plots, showing the ease with which the different signals can be classified. As discussed earlier, the patterns for each class are made to cluster around a point defined for that class in the decision space, and thus the closer the patterns are to the point, the easier it is to classify them. The boundary between the two classes under consideration in each plot is the diagonal line shown dotted. Each pattern that crosses the boundary line is essentially misclassified.

For ease of reference, the results of classification are plotted in tabular form, the confusion matrix, which shows the performance of the system at a glance. We represent the true classes of interest as the columns of the matrix, with the rows being the classes to which they are assigned. The results based on five features and listed in Table 4 show that 58 out of the 60 chip formation signals were classified as correct, a performance rate 

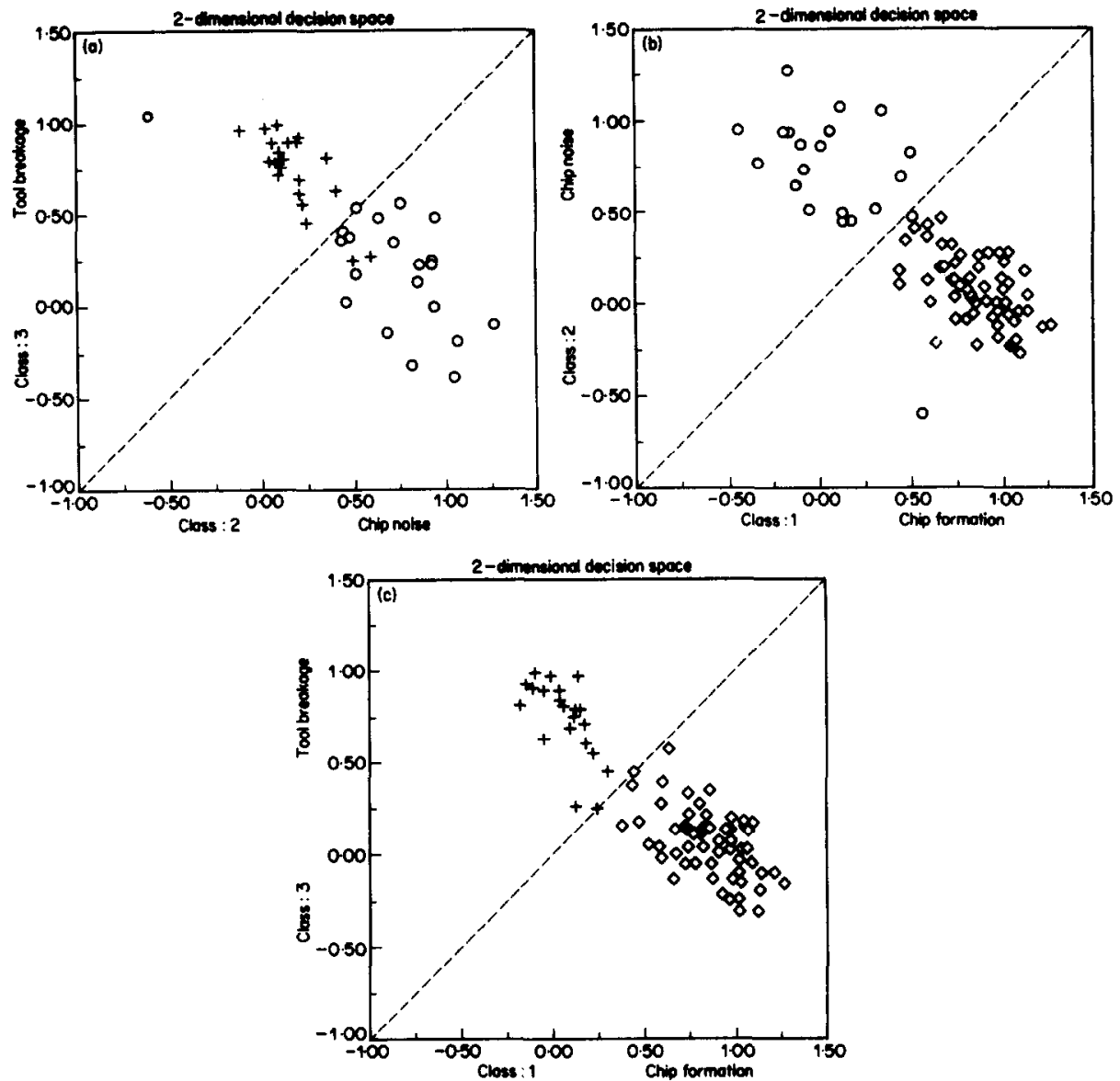

Figure 7. (a) Decision space plot for tool breakage-chip noise. (b) Decision space plot for chip noise-chip formation. (c) Decision space plot for chip formation-tool breakage. $\diamond$, Chip formation; $O$, chip noise; $t$, tool breakage.

TABle 4

Confusion matrix for five features

\begin{tabular}{lccc}
\hline & \multicolumn{3}{c}{ Input pattern } \\
\cline { 2 - 4 } $\begin{array}{c}\text { Output of } \\
\text { classifier }\end{array}$ & $\begin{array}{c}\text { Chip } \\
\text { formation }\end{array}$ & $\begin{array}{c}\text { Chip } \\
\text { noise }\end{array}$ & $\begin{array}{c}\text { Tool } \\
\text { breakage }\end{array}$ \\
\hline Chip formation & 58 & 1 & 0 \\
Chip noise & 1 & 18 & 2 \\
Tool breakage & 1 & 2 & 19 \\
Performance (\%) & 97 & 86 & 90 \\
\hline
\end{tabular}


$97 \%$. The corresponding rate for tool breakage was $90 \%$, with 19 out of the total of 21 being classified as correct, while two were classified as resulting from chip noise. The chip noise signals had the worst performance, with a classification rate of $86 \%$. Two out of the 21 signals were assigned to tool fracture, while another one was misclassified as resulting from chip formation. It is of interest to note that all the misclassified tool breakage signals were assigned to chip noise. This is probably due to similarities in the signals they generate, with both being burst type signals.

It has to be noted that the results obtained are applicable to the cutting conditions used, and as conditions are changed during machining, it will affect the system performance. Our objective until now has been to understand the system characteristics without the complex effects of the input variables. Incorporation of the cutting conditions in the design process is the subject of current research.

\section{CONCLUSIONS}

For monitoring the condition of the cutting tool in real time, acoustic emission signals generated during the cutting process have been analysed using pattern recognition techniques. The signals from the cutting process were considered as being generated by three different sources, viz., chip formation, tool fracture, and chip noise. A discriminant function was developed for each of these sources using the spectral components as features and formed the basis for classification. Five features were used in all, in the frequency range $100 \mathrm{kHz}$ to $1 \mathrm{MHz}$, with each feature consisting of a $20 \mathrm{kHz}$ bandwidth, and were selected using the class mean scatter criterion. The coefficients of the discriminant functions were obtained by training the system using signals generated by each of the sources of interest. An AISI 1018 steel was used for machining with a titanium carbide-coated cutting tool. Cutting speeds ranged from 200 to $800 \mathrm{ft} / \mathrm{min}(1$ to $4 \mathrm{~m} / \mathrm{s})$ with feed rates 0 f 0.0005 to $0.0075 \mathrm{in} / \mathrm{rev}(0.0133 \mathrm{~mm} / \mathrm{rev}$ to $0.191 \mathrm{~mm} / \mathrm{rev})$ and depth of cut 0.17 in $(4.32 \mathrm{~mm})$. The results show a classification rate of $90 \%$ for tool breakage, while those for chip formation and chip noise were 97 and $86 \%$ respectively.

\section{ACKNOWLEDGEMENT}

This work was supported by the University of Michigan Industrial Consortium on Diagnostic Sensing and Control for Metal Cutting; the Industrial Technology Institute; and the National Science Foundation under Grant Number DMC 8607198.

\section{REFERENCES}

1. N. H. Cook, K. Subramanian and S. A. Basile Survey of the State-of-the-Art of Tool Wear Sensing Techniques. MIT report prepared under NSF Grant No. FI-43861, 1975.

2. G. F. MiChELETTI, W. KOENIG and H. R. VICTOR 1976 Annals of the CIRP, 25, 483-496. In-process tool wear sensors for cutting operations.

3. J. TLuSTY and G. C. ANDREwS 1983 Annals of the CIRP, 32, 563-572. A critical review of sensors for unmanned machining.

4. S. K. BIRLA, 1981 Technology of Machine Tools. Sensors for adaptive control. LLNL: Livermore, CA.

5. K. IWATA and T. MORIWAKI 1977 Annals of the CIRP, 26, 21-26. An application of acoustic emission measurement to in-process sensing of tool wear.

6. I. GrabeC and P. LeSkovar 1977 Ultrasonics, January, 15, 17-20. Acoustic emission of a cutting process.

7. Y. KAKINO 1980 ASM Proceedings of an International Conference on Curting Tool Materials, pp. 25-39. In-process detection of tool breakage by monitoring acoustic emission. 
8. E. KANNATEY-ASIBU, JR. and D. A. DORNFELD 1981 Journal of Engineering for Industry, 103, 330-340. Quantitative relationships for acoustic emission from orthogonal metal cutting.

9. I. INASAKI and S. YONETSU 1981 Proceedings of The International Machine Tool Design and Research Conference, pp. 261-268. In-process detection of cutting tool damage by acoustic emission measurement.

10. E. KANNATEY-ASIBU, JR. 1982 Proceedings of the 10th NAMRC, pp. 487-492. On the application of the pattern recognition method to manufacturing process monitoring.

11. E. EMEL and E. KANNATEY-ASIBU, JR. 1986 Proceedings of the 14th NAMRC, pp. 266-272. Characterization of tool wear and breakage by pattern recognition analysis of acoustic emission signals.

12. D. A. DORNFEld and M. S. LAN 1983 Proceedings of the 11th NAMRC, pp. 386-389. Chip form detection using acoustic emission.

13. Y. Kakino, H. Suizu, M. Hashitai, T. Yamada, H. Yoshioka and A. Fujiwara 1983 Bulletin of the Japanese Society of Precision Engineering, 17, 241-246. In-process detection of thermal crack of cutting tool by making use of acoustic emission.

14. K. UEHARA and Y. KANDA 1984 Annals of the CIRP, 33, 71-74. Identification of chip formation mechanism through acoustic emission measurements.

15. D. A. DORNFELD, and C. S. PAN 1985 Proceedings of the 13th NAMRC, pp. 299-303. Determination of chip forming states using linear discriminant function technique with acoustic emission.

16. E. N. DiEI, and D. A. DORNFELD 1985 Sensors and Controls for Manufacturing, pp. 33-39. A model of tool fracture generated acoustic emission during machining. ASME.

17. N. AHMED, and K. R. RAO 1975 Orthogonal Transforms for Digital Signal Processing. New York: Springer.

18. D. H. FOLEY 1972 IEEE Transactions on Information Theory 18, 618-626. Considerations of sample and feature size.

\section{APPENDIX: NOMENCLATURE}

$C$ number of classes

$C_{i}$ label of $i$ th class

$\mathrm{g}_{i} \quad$ linear discriminant function for class $C_{i}$

$h$ height of rectangular window

$H$ Fourier transform of rectangular window

$M_{i}$ number of patterns in class $C_{i}$

$N$ number of sampled data values

$p_{i}$ a priori probability for class $C_{i}$

$\mathbf{Q}$ feature selection criterion

$\mathbf{R}_{\mathbf{i}}$ frequency domain covariance matrix for $C_{i}$

$\mathbf{S}_{i j}$ decision space data vector for $C_{i}$

$T$ transformation matrix

$\mathbf{V}_{i}$ predetermined cluster point in decision space

$w_{i j}$ weighting coefficient of the $k$-th feature in class $C_{i}$

$X$ time domain data vector

Y spectral domain feature vector

$\varepsilon_{\mathbf{i}} \quad$ mean square error vector for class $C_{i}$

$\theta$ threshold of discriminant function 